\title{
Modeling Heat and Mass Transfer During Evaporation in the Film Apparatus with Cross Movement Phases
}

\author{
Lukashov V.K. ${ }^{1}$, Kostiuchenko Y.V. ${ }^{1,2}$, Timofeev S.V. ${ }^{1}$ \\ 1. Shostka Institute of Sumy State University, UKRAINE, Shostka, 1 Haharina Str., 41100, E-mail: \\ khtms@ishostka.sumdu.edu.ua \\ 2. Sumy State University, UKRAINE, Sumy, 2 Rymskogo-Korsakova Str., 40007, E-mail: \\ y.kostiuchenko@pohnp.sumdu.edu.ua
}

\begin{abstract}
A mathematical model has been developed for heat and mass transfer in a solution film flowing down a heated surface under conditions of evaporation into a cross flow of a neutral (inert) gas. An experimental setup has been created and the coefficients of heat and mass transfer for an aqueous solution of glycerol, which are the parameters of the model, have been determined. Modeling heat and mass transfer made it possible to establish the regularities of changes in the temperature and concentration of the solution along the length of the film.
\end{abstract}

Keywords - heat and mass transfer, evaporation, falling film, cross flow, neutral gas.

\section{Introduction}

Apparatus with gravitational film flow of liquid are widely used in industry for various technological processes. In many cases, this flow is accompanied by evaporation from the free surface of the film. The presence of a neutral (inert) gas over the film has a significant effect on heat and mass transfer in this system [1]. For its direct and countercurrent movement, heat and mass transfer during evaporation from a liquid film has been well studied. For the cross interaction of these flows, there is almost no information about the study, which makes it difficult to calculate and model. At the same time, from the viewpoint of increasing the efficiency of technological devices, the cross interaction of flows is of interest [2]. The aim of the work was to establish the regularities of heat and mass transfer during evaporation in film devices with cross-phase movement.

\section{Mathematical Model}

Mathematical modeling in this case is reduced to solving a system that includes the equations: fluid motion in the film; film continuity; mass transfer from the free surface of the film to the gas phase; material balance of the gas phase for the evaporated substance; the relative content of the evaporated substance in the gas phase; Dalton's law; heat transfer in the film; heat transfer in the gas phase; material balance of the film in terms of the evaporated substance.

To solve this system of equations, the numerical method of the explicit Euler scheme was used. As a result, recurrent formulas were obtained for calculating the change in the temperature of the solution film, air temperature, and water concentration in the solution along the length of the film. The calculations took into account the dependence of the solution characteristics (density, specific heat capacity, viscosity) on these values. The coefficients of heat transfer from the heated surface to the film and from the film to the gas phase required for calculations, as well as the coefficient of mass transfer of evaporated water into the gas phase, were determined from the results of an experimental study.

\section{Research Methodology}

The experimental study of heat and mass transfer was carried out on the setup, which is a model of the working element of the film apparatus [2]. The main unit of the setup was a 
rectangular container, the outer surface of one of the vertical walls (working surface) of which, $0.8 \times 0.01 \mathrm{~m}$ in size served for the solution film to flow down. A high-temperature liquid coolant circulated through the container, which was in contact with the inner surface of this wall. A boundary wall is placed at a distance of $18 \mathrm{~mm}$ from this surface. Air was supplied to the space between this wall and the working surface, uniformly in height and perpendicular to the movement of the film. An aqueous solution of glycerin was used as a liquid. The setup is equipped with systems for regulating and measuring the flow rate and temperature of the solution entering from above and flowing down from the bottom of the working surface, the temperature of the coolant in the tank, the flow rate and temperature of the air entering and leaving the space between the working surface and the boundary wall. The concentration values of glycerol in the initial solution and the solution flowing down from the bottom of the working surface were determined by its density.

\section{Results and Discussion}

The simulation results are presented as graphs of temperature variation of the solution and the solute concentration in the film in the direction of its movement for different parameters of the evaporation process. As an example, graphs (fig. 1) of changes in solution temperature and glycerol concentration therein in the direction of film movement are given for different values of the initial solution consumption.
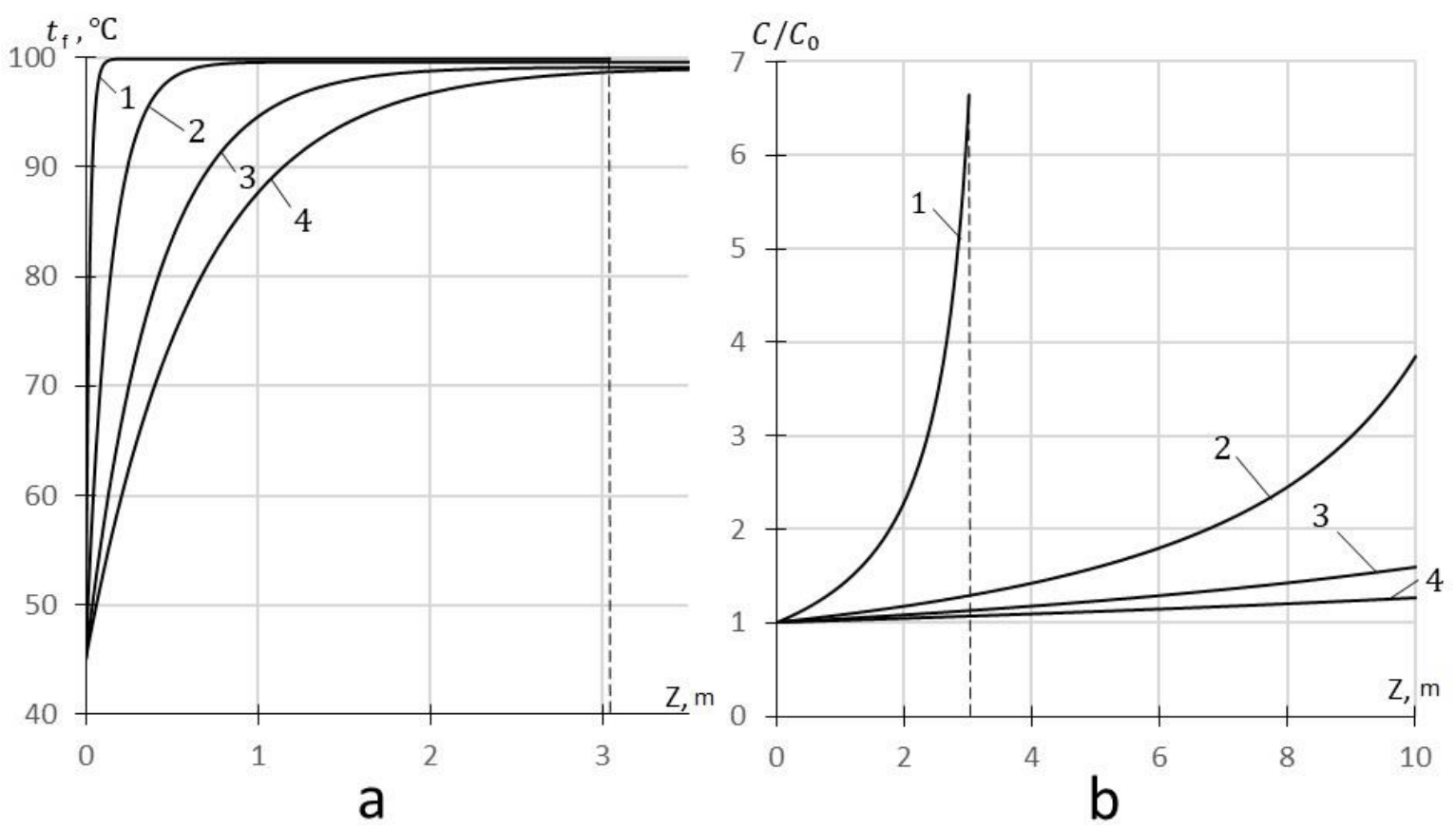

Figure 1 - Change in temperature (a) and concentration of glycerol (b) in its aqueous solution along the length of the film at different initial flow rates of the solution: $1-\mathrm{L}_{0}=0.00186 \mathrm{~kg} / \mathrm{s} ; 2-0,00485 \mathrm{~kg} / \mathrm{s} ; 3-0,00632 \mathrm{~kg} / \mathrm{s} ; 4-0,0102 \mathrm{~kg} / \mathrm{s}$

It follows from these graphs that the temperature of the solution in the film (fig. 1a) increases along its length and at a certain distance from the beginning reaches an equilibrium value, which remains constant until the water completely evaporates (curve 1 in fig. 1a). The intensity of the temperature rise along the length of the film increases with a decrease in the initial consumption of the solution. 
The change in the glycerol concentration in the solution (fig. 1b) at the beginning of the film is close to linear, then it sharply rises until the complete evaporation of water (curve 1 in fig. $1 \mathrm{~b}$ ). Comparison of these curves with the solution temperature distributions in the film suggests that their linear character at the beginning of the film is associated with the temperature reaching steady-state values. After reaching the steady-state temperature, an increase in the concentration of the solution along the length of the film is observed.

Also temperature changes of the solution and the concentration of glycerol in it along the length of the film from the initial temperature of the solution, the air velocity in the space above the film, its initial temperature and surface temperature along which film flows were established.

\section{Conclusions}

On the basis of the developed mathematical model and the performed experimental studies, the regularities of heat and mass transfer in a solution film flowing down a heated surface under the conditions of evaporation into a cross flow of a neutral gas were established. An example of changes in temperature and concentration of an aqueous solution of glycerol shows their growth in the direction of film movement. At a certain distance from the initial section of the film, the temperature reaches an equilibrium value, which increases with an increase in the flow rate of the solution. Changing the concentration at the beginning of the film is close to linear character, then when the temperature reaches an equilibrium value, it occurs a sharp rise, which lasts until complete evaporation of the water.

\section{References}

[1] Serafimov L.A., \& Frolkova A.V. (2008). Konovalov's first law validity in reactive distillation processes with inert, Fine Chemical Technologies, 3(2), 47-54.

[2] Lukashov, V.K., Romanko, S.M., Kostiuchenko, Y.V. (2019). Apparatus for concentrating a solution of sulfuric acid. Ukraine, No 134162. 